\title{
REVIEW ARTICLE OPEN Immune checkpoint pathways in immunotherapy for head and neck squamous cell carcinoma
}

\author{
Zi Mei ${ }^{1}$, Junwen Huang ${ }^{1}$, Bin Qiao $^{1}$ and Alfred King-yin Lam (iD ${ }^{1,2}$
}

With the understanding of the complex interaction between the tumour microenvironment and immunotherapy, there is increasing interest in the role of immune regulators in the treatment of head and neck squamous cell carcinoma (HNSCC). Activation of $T$ cells and immune checkpoint molecules is important for the immune response to cancers. Immune checkpoint molecules include cytotoxic T lymphocyte antigen 4 (CTLA-4), programmed death 1 (PD-1), T-cell immunoglobulin mucin protein 3 (TIM-3), lymphocyte activation gene 3 (LAG-3), T cell immunoglobin and immunoreceptor tyrosine-based inhibitory motif (TIGIT), glucocorticoid-induced tumour necrosis factor receptor (GITR) and V-domain lg suppressor of T cell activation (VISTA). Many clinical trials using checkpoint inhibitors, as both monotherapies and combination therapies, have been initiated targeting these immune checkpoint molecules. This review summarizes the functional mechanism and use of various immune checkpoint molecules in HNSCC, including monotherapies and combination therapies, and provides better treatment options for patients with HNSCC.

International Journal of Oral Science (2020)12:16

; https://doi.org/10.1038/s41368-020-0084-8

\section{INTRODUCTION}

According to the Global Cancer Report in 2018, head and neck squamous cell carcinoma (HNSCC) was the eighth most frequent cancer in the world, and the mortality rate ranked eighth among all cancers. ${ }^{1}$ Despite the trend of improved survival rates for patients with cancer over the past 20 years, local and distant failure after treatment of advanced HNSCC occur in up to $40 \%$ and $30 \%$ of patients, respectively. ${ }^{2}$

The past two decades have seen a surge in medical research surrounding cancer treatments, resulting in new strategies that have provided hope that we may effectively treat many cancers. We have improved our understanding of the pathology and molecular details of cancer and can perform gene sequencing based on individual cancer cells to obtain more "personalized treatment". Clinical trials of gene-targeting agents have successfully laid the foundation for other cancer therapies.

With the discovery of cancer immunotherapy and a deeper understanding of the $T$ cell responses to targeted immune checkpoint therapies, as well as the success of clinical trials of drugs that block immunological checkpoints, research predicting and identifying precise biomarkers of genomically targeted agents will become more popular. Because the immune response is dynamic and variable, identifying a useful drug in the field of cancer immunotherapy may involve more than just identifying a single biomarker to select a subset of patients for treatment. Therefore, we must evaluate the timeliness of the immune response and determine the effect of the immune response on the clinical treatment to achieve the best therapeutic effect with combination treatments (Fig. 1).

HNSCC tissues are in an immunosuppressive state, with fewer lymphocytes, natural killer (NK) cells and specific antigens than normal tissues. ${ }^{3}$ Thus, this cancer type is called a "cold tumour". HNSCC can escape immune surveillance through different mechanisms.

Immunological checkpoint inhibitors, which target $T$ cell regulatory pathways to enhance antitumour immune efficiency, have brought great clinical advances and offer new methods for anticancer treatment. In addition to surgery, radiotherapy and chemotherapy, targeting immune checkpoints has become another good choice for clinicians to treat HNSCC. To date, the U.S. Food and Drug Administration (FDA) has approved many immune checkpoint drugs for clinical usage.

Tumour microenvironment of HNSCC

The immune system plays a key role in the formation, development, and progression of HNSCC. There are more infiltrating regulatory $T$ cells (Tregs) in patients with human papillomaviruspositive $\left(\mathrm{HPV}^{+}\right)$HNSCC than in those with $\mathrm{HPV}^{-} \mathrm{HNSCC}$. Studies have shown that high levels of cluster of differentiation (CD) $8^{+}$ tumour-infiltrating lymphocytes (TILs) in $\mathrm{HPV}^{+} \mathrm{HNSCC}$ tissues are associated with improved disease-free survival. ${ }^{4}$ Primed effector $\mathrm{CD}^{+}$cytotoxic T lymphocytes (CTLs) can exit the lymphoid tissue, move towards their target antigens, and identify such antigens in the context of major histocompatibility complex (MHC) class I molecules for target cell lysis. In addition, the number of $\mathrm{CD}^{+} \mathrm{T}$ helper (Th1) lymphocytes has a significant correlation with $\mathrm{CD}^{+}$ $\mathrm{T}$ cell density in the tumour microenvironment. ${ }^{5}$ In peripheral blood, T helper 17 (Th17) and T cytotoxic 17 (Tc17) cells were significant predictors of poor prognosis for patients with HNSCC. ${ }^{6}$

Mechanism of immune escape in HNSCC

Immunological checkpoints are immunosuppressive molecules that can maintain self-tolerance by modulating $T$ cell function and

\footnotetext{
${ }^{1}$ Department of Oral and Maxillofacial Surgery, The First Affiliated Hospital of Zhengzhou University, Zhengzhou, China and ${ }^{2}$ Cancer Molecular Pathology and Griffith Medical School, Griffith University, Gold Coast, Queensland, Australia

Correspondence: Bin Qiao (qiaobin@zzu.edu.cn) or Alfred King-yin Lam (a.lam@griffith.edu.au)

These authors contributed equally: Zi Mei, Junwen Huang
}

Received: 2 January 2020 Revised: 17 April 2020 Accepted: 29 April 2020

Published online: 28 May 2020 


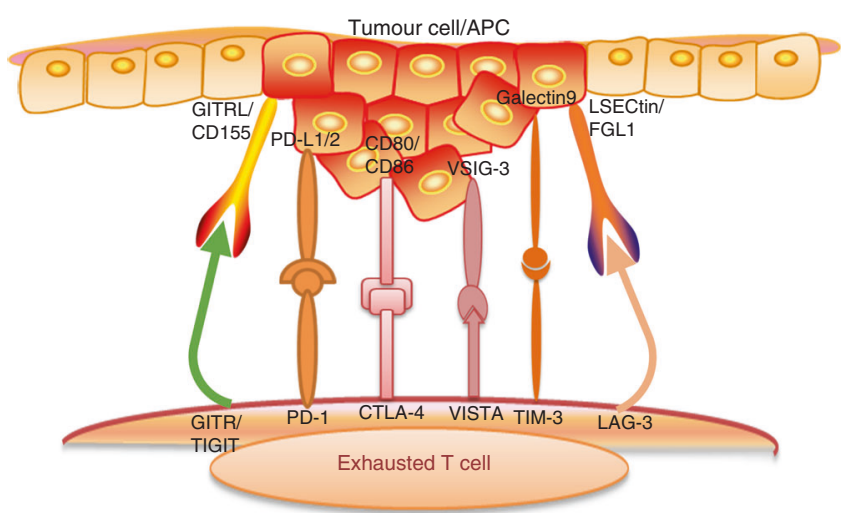

Fig. 1 The coinhibitory pathways of head and neck squamous cell carcinoma. During carcinogenesis, T cell activation is inhibited by a number of pathways, which are often activated by the expression of certain ligands on tumour cells or antigen-presenting cells (PD-L1/2, CD80/CD86, galectin 9, LSECtin/FGL1, CD155/GITRL, and VSIG-3) that bind to receptors on T cells (PD-1, CTLA-4, TIM-3, LAG-3, TIGIT, GITR, and VISTA) and inhibit the activation and the anti-tumour function of T cells

protecting surrounding tissues by suppressing immune responses. Tumour cells can take advantage of this feature to evade attack by immune cells. In patients with HNSCC, immune checkpoints are the basic mechanism leading to cancer cell escape. To eliminate HNSCC using immunotherapy, specific identification of tumour cells and lysis by CTLs are required. The human lymphocyte antigen (HLA) system is complex and polymorphic, and is the human version of the major histocompatibility complex (MHC) system. HLA molecules are mainly present on the surface of antigen-presenting cells (APCs) and present tumour antigen peptides to CTLs. ${ }^{7}$ By altering the expression of HLA-I and impairing antigen processing machinery (APM), HNSCC cells can reduce tumour antigen recognition by $T$ cells. Through the sequencing of HNSCC cells, multiple mutations have been detected in HLA alleles and antigen processing machinery components. Chromosome and regulatory defects related to genes encoding HLA and APM factors can lead to the selective deletion of HLA and APM components in a large number of HNSCCs and are associated with a worse prognosis of patients. ${ }^{8}$

Tumour cells with complete loss of HLA can escape the immune response by evading recognition by $T$ cells. However, this can trigger the activation of NK cells because the loss of HLA can eliminate suppression of NK cell activation. ${ }^{9}$ Therefore, tumour cells can use a variety of mechanisms to achieve immune evasion while avoiding complete loss of HLA expression. ${ }^{10}$

In summary, downregulation or deletion of HLA class I molecules or APM components is one of the strategies by which tumours evade the immune response. In addition, tumour cells secrete immunosuppressive and pro-apoptotic factors, as well as upregulate inhibitory cell surface molecules that can lead to evasion of the immune response.

\section{THE BASIC BIOLOGY OF IMMUNE CHECKPOINTS}

Activation and regulation of $T$ cells

$T$ cell activation requires dual signalling: the first signal is induced when the $T$ cell receptor (TCR) recognizes the MHC-antigen; the second signal is mediated by the B7 molecule on the surface of the APC and the CD28 molecule on the surface of the T cell, which are also known as co-stimulatory factors. Without either of the two signals, T cells cannot be activated. Importantly, the initiation and activation of $\mathrm{T}$ cells are regulated by central and peripheral checkpoints. ${ }^{11}$ Activation of T cells is bidirectional; in addition to initiating proliferation and differentiation, $\mathrm{T}$ cell activation also induces an inhibitory pathway that ultimately attenuates and terminates $\mathrm{T}$ cell responses.

Function of immune checkpoints in HNSCC

An immune checkpoint is a regulatory signal that affects immune activation and self-tolerance. Immune checkpoint signalling is critical for preventing autoimmunity and protecting host tissues from immune-mediated indirect damage (Fig. 1).

Cytotoxic $T$ lymphocyte antigen 4 (CTLA-4). As the first clinically targeted immune checkpoint receptor, CTLA-4 is expressed primarily in $T$ cells and is less expressed in active $B$ cells, monocytes, granulocytes and dendritic cells (DCs). ${ }^{12}$ CTLA-4 is also expressed on Tregs and produces the immunosuppressive molecule transforming growth factor- $\beta$ (TGF- $\beta$ ) when activated with CD28. ${ }^{13}$ Both CTLA-4 and CD28 act as transmembrane receptors. CTLA-4 can bind the B7 protein to induce $T$ cell dysfunction and participate in negative regulation of the immune response. Under normal circumstances, the immunosuppressive effect of CTLA-4 is to stimulate the immune response effectively without excessive damage to normal tissues. However, cancer cells secrete TGF- $\beta$, which can stimulate the expression of CTLA-4, leading to $\mathrm{T}$ cell exhaustion. ${ }^{14} \mathrm{~T}$ cell exhaustion is a state in which $T$ cells have weak functions and might exert immunosuppression. The affinity of CD28 for CTLA-4 on the surface of T cells exceeds its affinity for the co-stimulatory molecules CD80 and CD86. Thus, $T$ cells are prevented from proliferating and fail to function. ${ }^{15}$

Both CTLA-4 and CD28 bind to the two ligands CD80 and CD86, which are expressed on the surface of antigen-presenting cells. In addition, activation of T cells also leads to the expression of CTLA4 , which aggregates in T cells at the T cell-antigen-presenting cell (APC) interface, reaching a level that ultimately prevents costimulatory stimulation and abolishes activated $T$ cell responses. In addition, CTLA-4 is also expressed on Tregs. CTLA-4 is contains properties that cause it to sometimes localize to plasma membranes. Approximately $90 \%$ of CTLA-4 is intracellular. CTLA4 also differs from $\mathrm{CD} 28$ in that it causes a high degree of endocytosis, with more than $80 \%$ of surface molecules being internalized within $5 \mathrm{~min}$. In addition, researchers found that CTLA- 4 could be released from the endosomes once internalized, and then the endosomes could be recycled to the plasma membrane. We observed limited colocalization of CTLA-4 with lysosomes in labelling experiments, yet other experiments have shown that CTLA-4 degradation can be inhibited by lysosomal blockade. $^{16}$

Programmed cell death protein-1/programmed death-ligand 1 (PD$1 / P D-L 1)$. PD-1 belongs to the CD28 receptor family and is mainly expressed on activated T cells and B cells. It is also found in monocytes and a small fraction of thymocytes. The ligands for PD1 include PD-L1 and PD-L2. Both ligands are expressed on endothelial and epithelial antigen-presenting cells and activated lymphocytes. ${ }^{17}$ Although PD-L1 has limited expression in normal tissues, it is inducible and constitutively expressed in many solid and haematological malignancies. PD-L1 overexpression in tumour cells can promote tumour formation. ${ }^{18}$ In non-small-cell lung carcinoma (NSCLC) and melanoma, high expression of PD-L1 on tumour cells is strongly associated with high tumour grade and poor prognosis of patients. ${ }^{19,20}$ HNSCC tissues produce PD-L1 through an abnormal PD-1 signalling pathway, which leads to tumour immunosuppression. ${ }^{21}$ The PD-1/PD-L1 signalling pathway can be activated in a chronic inflammatory environment. In fact, the formation of $\mathrm{HPV}^{+} \mathrm{HNSCC}$ is mainly due to the activation assisted by the PD-1/PD-L1 axis. HPV ${ }^{+}$HNSCC tissues had more lymphocytes and higher levels of PD-L1 than HPV HNSCC tissues, and the infiltrated CTLs expressed more PD-1. ${ }^{22}$ PD-L1 can deliver additional immunosuppressive signals by binding to the CD80 receptor, which is expressed on T cells. Thus, depending on the 
immune environment within different HNSCC tumour tissues, the PD-1/PD-L1 axis can be blocked at different levels: targeting PD-1 can block its binding to PD-L1/PD-L2, whereas targeting PD-L1 can inhibit its binding to PD-1/CD80.

Interactions between PD-L1 and PD-1 can directly regulate the tumour microenvironment and have different functional significance in modulating the effects of T cells, DCs, bone marrowderived suppressor cells (MDSCs) and Tregs. Blocking PD-1 on Tregs inhibits their ability to mediate immune tolerance, but this does not indicate that PD-1 can directly enhance Treg function. ${ }^{23}$ On effector T cells, PD-1 expression is considered an exhausted marker, and it also increases sensitivity to the PD-L1 death signalling pathway. The PD-L1 signalling pathway not only downregulates antitumour $T$ cell function but also affects the cellular interactions between the innate immune response and the adaptive immune response, such as interactions between DCs, MDSCs and Tregs.

Because PD-1 and CTLA-4 belong to the same protein family and have similar structural fragments, Freeman and his colleagues speculated that PD-L1 is part of the B7 family. ${ }^{24}$ Inflammatory cytokines can induce PD-L1 expression, for example, type I and type II interferon (IFN), tumour necrosis factor-alpha (TNF-a) and vascular endothelial growth factor (VEGF). The PD-L2 molecule is only expressed on activated macrophages and dendritic cells. Once PD-L1 binds to PD-1, it will inhibit T cell receptor-mediated lymphocyte proliferation and cytokine secretion. In activated T cells, PD-1 binds to PD-L1 and then promotes phosphorylation of tyrosine in the immunoreceptor tyrosine-based switch motif (ITSM) domain of PD-1, which leads to dephosphorylation of downstream protein kinases, spleen tyrosine kinase (Syk) and phosphatidylinositol 3-kinase (PI3K), and inhibition of downstream protein kinase $B$ (AKT) and extracellular regulated protein kinase (ERK). The activation of ion channels ultimately inhibits the transcription and translation of genes and cytokines required for $T$ cell activation and plays a role in negatively regulating $T$ cell activity.

T cell immunoglobulin mucin-3 (TIM-3). As a member of the TIM family, TIM-3 was first reported in 2002 and was specifically expressed on $\mathrm{CD}^{+}{ }^{+}$Th1 but not Th2 lymphocytes. ${ }^{25}$ It is also expressed on Tregs, DCs, monocytes, mast cells, NK cells and TILs. TIM-3 is also found on tumour cells, such as melanoma and B cell lymphoma cells. ${ }^{26}$ Studies have shown that TIM-3 and its ligands can regulate T cell tolerance. Galectin- 9 has been identified as a major ligand for TIM-3 and is a member of the galectin family. ${ }^{27}$ It can regulate diverse biological functions of tumour cells, aggregation, adhesion and apoptosis. ${ }^{28}$ When TIM-3 binds to its ligand galectin-9, it can inhibit the expansion of Th1 and Th17 cells. This change will promote apoptosis of Th1 cells, deplete the function of $\mathrm{CD}^{+}$T cells, induce massive expansion of MDSCs and hamper the immune response. In the early stage of tumour formation, $\mathrm{TIM}-3^{+} \mathrm{CD} 4^{+}$T cells can secrete IFN- $\gamma$ to have antitumor effects. On the other hand, in the middle and late stages of tumour formation, TIM $-3^{+}$Tregs proliferate, thereby blocking the function of effector T cells. ${ }^{29}$ When TIM-3 was blocked with an anti-TIM-3 monoclonal antibody, the antitumor response of T cells mediated by IFN- $\gamma$ was promoted. ${ }^{29}$

The expression of TIM-3 is positively correlated with the survival of pancreatic ductal adenocarcinoma and renal cell carcinoma. ${ }^{30,31}$ However, The Cancer Genome Atlas (TCGA) database showed that the expression of TIM-3 is not associated with patient pathological tumour node metastasis (TNM) stage in HNSCC. On the other hand, TIM-3 expression has a positive relationship with the presence of lymph node metastasis and recurrence. ${ }^{32}$ Elevated expression of TIM-3 contributes to the exhaustion of effector $\mathrm{T}$ cells, which may lead to ineffective antitumour immune response and tumour clearance, resulting in cancer metastasis and recurrence in patients with HNSCC. ${ }^{33}$ When treatment with
anti-TIM-3 antibodies was used in an HNSCC mouse model, the $\mathrm{CD} 4^{+} \mathrm{TIM}^{-} 3^{+}$to $\mathrm{CD} 8^{+} \mathrm{TIM}-3^{+}$ratio was affected, which led to an increase in the function of $\mathrm{CD}^{+}$and $\mathrm{CD}^{+} \mathrm{T}$ cells and a decrease in marrow-derived suppressor cells, significantly inhibiting tumorigenesis and improving antitumour immune responses. ${ }^{32}$

In autoimmune diseases, the expression of TIM-3 is increased in macrophages. M2 macrophages are alternatively activated (in contrast to M1 macrophages) by exposure to certain cytokines. Increased expression of TIM-3 in M2 macrophages participates in immune regulation by inhibiting macrophage activation. ${ }^{31}$ Another study also showed that upregulation of TIM-3 expression in mouse M2 macrophages could mediate inflammatory responses. $^{34}$

Lymphocyte activated gene-3 (LAG-3). The surface molecule LAG$3 / C D 223$, a member of the immunoglobulin superfamily, was first discovered in $1990 .{ }^{35}$ It is mainly expressed on activated T cells and can be expressed on NK cells, B cells and plasmacytoid dendritic cells. LAG-3 negatively modulates $T$ cell proliferation, activation and homeostasis ${ }^{36}$ and has similar functions to CTLA-4 and PD-1. ${ }^{37}$ LAG-3 plays a vital role in the inhibitory function of Tregs. ${ }^{38}$ In some malignancies, the coexpression of LAG-3 and PD-1 on tumour-infiltrating lymphocytes is related to the impaired function of $\mathrm{CD}^{+}$effector T cells, which promotes the immune escape of tumours. ${ }^{39}$ LAG-3 has a similar protein sequence to the CD4 receptor. One of its ligands is an MHC-II molecule, and LAG-3 has an even stronger affinity for this ligand than CD4. ${ }^{40}$ In fact, the effect of LAG-3 on CD8 ${ }^{+}$T cell function does not depend on the interaction with MHC-II; liver and lymph node sinusoidal endothelial cell C-type lectin (LSECtin) of the DC signalling family is considered to be another ligand for LAG-3. LSECtin binds to LAG-3 to inhibit the secretion of IFN- $\gamma$ by effector $T$ cells in melanoma, thereby inhibiting the antitumour immune response. ${ }^{41}$ Blocking LAG-3 leads to increased accumulation and effector function of $\mathrm{CD}^{+}{ }^{+}$T cells in ovarian cancer. ${ }^{42,43}$

LAG-3, generally expressed on Tregs, is required for Treg cellmediated $\mathrm{T}$ cell homeostasis. Blocking LAG-3 can inhibit Treg activation and abolish Treg inhibition, while the ectopic expression of LAG-3 by non-Treg $\mathrm{CD}^{+}{ }^{+} \mathrm{T}$ cells contributes to inhibitory activity. LAG-3 is further expressed on $\mathrm{CD}^{+}{ }^{+}$Foxp $3^{+}$, interleukin-10 (IL-10)-secreting type 1 regulated (Tr1) T cells. LAG-3 also has high expression on Tregs in HNSCC. ${ }^{44}$

In 2019, Wang and his colleagues found that fibrinogen-like protein 1 (FGL1), a liver-secreted protein, is a new ligand for LAG-3. ${ }^{45}$ FGL1 is the main LAG-3 functional ligand that functions independently of MHC-II. FGL1 inhibited the activation of antigenspecific T cells, and the ablation of mouse FGL1 promoted T cell immunity. Under normal physiological conditions, FGL1 is mainly secreted by liver cells and is involved in mitosis and metabolism. ${ }^{46}$ Blocking the FGL1-LAG-3 interaction with gene ablation or monoclonal antibodies enhances the $\mathrm{T}$ cell response and promotes antitumour immunity. FGL1 is upregulated in most human cancers and is associated with poor prognosis and treatment outcomes. The FGL1/LAG-3 pathway can be used as a potential target for immune escape mechanisms and cancer immunotherapy.

$T$ cell immunoglobulin and immunoreceptor tyrosine-based inhibitory motif (TIGIT). TIGIT is a member of the poliovirus receptor (PVR)/Nectin family, and is also known as Washington University cell adhesion molecule (WUCAM), V-set and transmembrane domain-containing 3 (Vstm3), V-set and immunoglobulin domain-containing 9 (VSIG9). It is mainly composed of four parts, namely, the extracellular immunoglobulin variable region (IgV) domain, a type 1 transmembrane domain, a classical ITIM and an immunoglobulin tyrosine tail (ITT) motif. TIGIT is expressed in lymphocytes, particularly in effector and regulatory $\mathrm{CD}^{+} \mathrm{T}$ cells, follicular helper $C D 4^{+} \mathrm{T}$ cells, effective $\mathrm{CD}^{+} \mathrm{T}$ cells and NK cells. ${ }^{4}$ 
CD155 (PVR, Necl5 or Tage4) is a high-affinity ligand of TIGIT. It is also a cell surface receptor that is highly expressed on endothelial cells, fibroblasts, DCs and tumour cells. ${ }^{48}$ CD155 is highly expressed on the surface of tumours, binds to TIGIT on the surface of NK and T cells, increases IL-10 secretion, reduces the secretion of pro-inflammatory cytokines, and inhibits the antitumour immune response. CD112 (PVRL2 or nectin 2) and CD113 (PVRL3) also bind with TIGIT, but their affinity for TIGIT is weaker than that of CD155.

CD155, CD112, and CD113 share a ligand with CD226 (DNAM-1). The inhibition of TIGIT can counteract the activation of the costimulatory molecule CD226, and TIGIT/CD226 forms a network that regulates human $T$ cell function, such as the CD28/CTLA-4CD80/CD86 pathway. Coactivation receptors are expressed on naive and resting $T$ cells, and corepression receptors are expressed after T cell activation. CD226 transmits a positive signal, and TIGIT transmits an inhibitory signal. ${ }^{49}$

Glucocorticoid induced TNFR family related gene (GITR). GITR is a new addition to the tumour necrosis factor receptor (TNFR) superfamily and is expressed on the surface of $\mathrm{CD} 25^{+} \mathrm{CD} 4^{+}$Tregs, effector T cells, and natural killer cells. ${ }^{50}$ Binding of GITR and its ligand GITRL can reduce the recruitment of Tregs, weaken their inhibitory function and activate the MAPK (mitogen-activated protein kinase)/ERK pathway and NF-KB signalling, increasing T cell proliferation, promoting secretion of pro-inflammatory cytokines, and enhancing antitumour function. ${ }^{51,52}$ Therefore, GITR is an immune checkpoint that can potentially be monitored after immunotherapy with an anti-GITR antibody (clone DTA-1) that blocks the inhibition of regulatory T cells. ${ }^{53}$

$V$-domain Ig suppressor of $T$ cell activation (VISTA). A newer checkpoint molecule, VISTA, also named differentiation of embryonic stem cells 1 (Dies1), Gi24, and PD-1 homologue (PD$1 \mathrm{H})$, is similar in function to PD-L1 and can effectively inhibit T cell activation. In mice, VISTA is highly expressed on tumourinfiltrating leukocytes, while blockade enhances antitumour immunity in multiple tumour models. ${ }^{54}$ VISTA is mainly expressed on myeloid APCs and T cells, especially on Tregs. ${ }^{55}$ VISTA helps created an immunosuppressed tumour microenvironment by enhancing Treg maturation and inhibiting T cell activation. VISTA and $\mathrm{PD}-1$ inhibit $\mathrm{T}$ cells through a non-redundant immune regulatory network and comodulate $T$ cell responses. ${ }^{56}$

VSIG3 (V-set and immunoglobulin domain-containing 3 ) is a novel ligand for VISTA, ${ }^{57}$ also known as immunoglobulin superfamily member 11 (IGSF11) and brain and testicular-specific immunoglobulin superfamily 11 (BT-IgSF), that mediates homogenic adhesion in a calcium-independent manner. ${ }^{58}$ The interaction of VSIG3 and VISTA on activated T cells inhibits $T$ cell proliferation and the production of cytokines and chemokines. The suppressive effect of VSIG3 on activated T cells and the high expression of VSIG3 in colorectal adenocarcinoma, hepatocellular carcinoma, and intestinal-type gastric carcinoma suggest that blocking the VSIG3/VISTA pathway could be a new cancer immunotherapy strategy. ${ }^{57}$

\section{CLINICAL TRIALS OF IMMUNE CHECKPOINT-BLOCKING THERAPEUTICS IN HNSCC \\ PD-1/PD-L1}

Currently, monoclonal antibodies against humanized PD-1 and PD-L1 are being used in clinical trials for patients with advanced solid tumours under the approval of the FDA. ${ }^{59}$ Among them, immune checkpoint inhibitors targeting the PD-1/PD-L1 axis have been approved for clinical usage in some malignancies, including non-small-cell lung carcinoma, urothelial carcinoma, renal cell carcinoma, Merkle cell carcinoma and hepatocellular carcinoma. ${ }^{60}$ There are four approved monoclonal antibodies targeting PD-1 and PD-L1, namely, nivolumab (opdivo), pembrolizumab (keytruda), avelumab (bavencio) and atezolizumab (tecentriq). The first two are anti-PD-1 antibodies, and the last two are anti-PD-L1 antibodies. Nivolumab and pembrolizumab have been approved by the FDA for patients with HNSCC with relapse or metastasis who are cisplatin-resistant. ${ }^{61}$

Nivolumab is an anti-PD-1 monoclonal lgG4 antibody that blocks co-suppression signals via the PD-1/PD-L1 axis. It became the first immunotherapy approved by the FDA for patients with HNSCC based on a phase III clinical trial (Checkmate 141, NCT02105636) on November 10, 2016. ${ }^{62}$ The study randomly selected 361 patients with recurrent HNSCC who progressed six months after platinum chemotherapy. The patients were allocated at a 2:1 ratio to receive either nivolumab (at a dose of $3 \mathrm{mg} \cdot \mathrm{kg}^{-1}$ of body weight) or standard single-drug systemic therapy every two weeks, such as methotrexate, docetaxel or cetuximab. The primary monitoring point was overall survival (OS) but objective response, progression-free survival (PFS), safety and patient-reported quality of life were also analysed. ${ }^{63}$ The results showed that the median survival of the group treated with nivolumab was 7.5 months, while the survival of the standard treatment group was 5.1 months (95\% confidence interval $[\mathrm{Cl}], P=0.01$ ). The estimated 1-year survival rates of the nivolumab and standard treatment groups were approximately $36.0 \%$ and $16.6 \%$, respectively. The PFS rate of the nivolumab grpi[at 6 months was $19.7 \%$, compared with $9.9 \%$ for the standard treatment group. In addition, the nivolumab group had fewer severe toxic effects (grade III or IV) than the standard treatment group (13.1\% vs $35.1 \%)$.

Patients treated with nivolumab appeared to have longer OS than patients receiving standard treatment, regardless of the expression levels of PD-L1 or the p16 status of the cancer. There was no significant relationship between the levels of PD-L1 (high versus low) and p16 (positive versus negative) in tumours regardless of treatment type.

Pembrolizumab is a high-affinity, humanised, IgG4-k monoclonal antibody targeting PD- 1 that was first approved by the FDA in 2017 according to the results of the phase Ib KEYNOTE 012 cohort amplification trial. ${ }^{64}$ In 2019, a phase III clinical study using pembrolizumab (KEYNOTE 048) in the treatment of relapsed or metastatic HNSCC reported superior treatment results from pembrolizumab. In the interim analysis, pembrolizumab combined with chemotherapy improved the OS in the general population compared with cetuximab combined with chemotherapy (13.0 months vs 10.7 months, $P=0.0034$ ). Based on the observed efficacy and safety results, pembrolizumab and chemotherapy are now the first-line treatment for patients with recurrent or metastatic HNSCC, whereas pembrolizumab monotherapy is the first-line treatment for patients with relapsed or metastatic PD-L1positive HNSCC. $^{65}$

Clinical trials have shown conflicting results. There are clinical responses in patients with $\mathrm{PD}-\mathrm{L}^{-}$cancer, whereas some patients with PD-L1 expression are resistant to immunotherapy. Therefore, the relationship of PD-L1 status with disease outcomes and the appropriate cut-off for PD-L1 expression need to be determined. PD-L1 immunohistochemical staining results are not always reliable enough to accurately predict the response to immunotherapy, and other biomarkers may be required. Qualitative and quantitative multiparametric analyses are helpful. Although the expression of PD-L1 is a main predictor, it must be balanced with other markers, such as the density, composition and activation state of inflammatory cells in the tumour microenvironment. ${ }^{66}$ The combination of the expression of PD-L1 in HNSCC tumour cells and the presence of CTLs is more predictive of tumour cell response than single expression in tumour cells. ${ }^{67}$

Both nivolumab and pembrolizumab are monoclonal antibodies that block PD-1. Durvalumab (Imfinzi; Medimmune/AstraZeneca) is another fully human monoclonal antibody that blocks the binding of PD-L1 to the receptors PD-1 and CD80. In the HAWK phase II 
study, it was used in patients with recurrent and/or metastatic HNSCC in whom PD-L1 expression was found in more than $25 \%$ of tumour cells after the failure of platinum-based chemotherapy. ${ }^{68}$

At the same time, another ligand, PD-L2, is receiving more attention. Researchers evaluated PD-L2 expression in tumour tissue using immunohistochemistry. In addition, the relationship between clinical response and PD-L2 status in human tumour tissues from patients with recurrent or metastatic HNSCC treated with pembrolizumab was also evaluated. The results showed that the levels of PD-L2 and PD-L1 in the tumour had a very significant relationship $(P<0.0001) .{ }^{69}$ PD-L2 expression can also be detected in tumours with no PD-L1 expression. This indicates the predictive power of PD-L2 is independent of PD-L1. ${ }^{70}$ Patients with PD-L1 ${ }^{+}$ and $\mathrm{PD}-\mathrm{L} 2^{+}$tumours $(27.5 \%)$ had a stronger response than those with only PD-L1 ${ }^{+}$tumours (11.4\%). Patients with PD-L2 ${ }^{+}$tumours have a longer median PFS and longer overall survival than patients with PD-L2 tumours.

Patients with tumour cells with simultaneous high levels of PD-1 in TILs and PD-L1 may have shorter disease-free survival than patients with single expression of either marker. ${ }^{71}$ These data highlight that PD-1/PD-L1 overexpression in cancer could have adverse outcomes. ${ }^{72}$ The microenvironment of HNSCC requires further exploration and understanding using a multiparametric approach. Some ongoing clinical trials are shown in supplementary Table 1.

CTLA-4

Since CTLA-4 can bind to B7, it prevents the interaction between B7 and the co-stimulatory molecule CD28 and restricts the proliferation of $\mathrm{T}$ cells and the production of $\mathrm{IL}-2 .^{73}$ Blocking CTLA-4 can abolish the inhibition of $T$ cells, leading to an antitumour immune response in the host. There are currently two human anti-CTLA-4 antibodies used in phase III clinical trials, ipilimumab and tremelimumab. Phase I/II studies have shown that both antibodies are safe and show some activity as monotherapy or when combined with IL-2 or conventional chemotherapy. ${ }^{74,75}$

In non-small-cell lung carcinoma or small-cell lung carcinoma, a large phase II clinical study of patients found that ipilimumab increased overall PFS and immune-related PFS. This indicated the unique response characteristics of ipilimumab, but the OS did not increase. $^{76}$ In addition, patients receiving ipilimumab after chemotherapy had the greatest increase in PFS, indicating that ipilimumab was most effective after chemotherapy-induced tumour antigen release. ${ }^{77}$ Tremelimumab could be used as second-line or third-line treatment in patients with relapsed malignant mesothelioma. These data suggest that ipilimumab may be promising for HNSCC, but researchers should be aware of the unique properties of the drug when considering treating patients with the therapy. More clinical trials are being used in combination with other drugs or treatments (nivolumab, relatlimab or cetuximab; NCT04080804).

\section{GITR}

AMG 228 is an agonistic human lgG1 monoclonal antibody that binds to human GITR. In one study, the investigators selected 30 patients (over 18 years old) with recurrent malignant solid tumours who met the experimental requirements (NCT02437916), including patients with non-small-cell lung carcinoma, HNSCC, melanoma and colon adenocarcinoma. AMG 228 was administered to patients intravenously every three weeks and was divided into two phase escalation doses: a single patient cohort $(3,9,30$, and $90 \mathrm{mg})$ followed by a "rolling 6" design ( $n=2-6 ; 180,360,600,900$ and $1200 \mathrm{mg}$ ). Finally, drug safety, pharmacokinetics, dose-limiting toxicity (DLT), pharmacodynamics, and maximum tolerated dose (MTD) were assessed based on the patients' response. The results showed that the drug was well tolerated. Patients did not show DLT, and the maximum administered dose did not reach the MTD. There was no evidence of T cell activation or antitumour activity with a single application of AMG 228 therapy. $^{78}$

TIM-3

Kim et al. found that TIM-3 blockade alone cannot significantly improve the overall survival rate of in vitro models, nor can it benefit the effector cell population. However, it increases the expression of inflammatory cytokines such as IFN- $\gamma$, TNF- $a$ and IL17a in tumours. In addition, they identified that TIM-3 inhibition can increase $\mathrm{CD}^{+}{ }^{+} \mathrm{T}$ cell dependence, as $\mathrm{CD}^{+}{ }^{+} \mathrm{T}$ cell depletion eliminates the anticancer effect of TIM-3 inhibition. ${ }^{79}$ Clinical trials are currently evaluating the role of combined blockade of TIM-3 and PD-1/PD-L1 in various advanced solid tumours (NCT02817633).

LAG-3

In a phase I/lla study, the anti-LAG-3 antibody BMS-986016 was used in combination with nivolumab (NCT01968109) in patients with relapsed melanoma treated with anti-PD-1 antibodies. In this trial, patients with melanoma must have had prior anti-PD-1/PD-L1 antibody ( \pm anti-CTLA-4 antibodies or BRAF/MEK inhibitors) treatment and progressive disease. These patients $(n=43)$ received BMS-986016 (80 $\mathrm{mg})$ and nivolumab $(240 \mathrm{mg})$. The results showed that $70 \%$ of these patients $(n=30)$ had prior anti-CTLA-4 antibody therapy, $47 \%(n=20)$ had $\geq 3$ prior therapies, and $35 \%(n=15)$ had proto-oncogene B-Raf and $v$-Raf murine sarcoma viral oncogene homologue $B(B R A F)$ mutations. Of the 31 patients who were effectively evaluated, the objective response rate $(O C R)$ was $16 \%$, and the disease control rate (DCR) was $45 \%$, with efficacy observed even in some patients who did not respond to previous anti-PD-1 therapy. ${ }^{80}$

\section{TIGIT}

TIGIT-targeting therapy is still in the early stages of clinical development. The antagonistic anti-TIGIT antibody OMP-313M32 (NCT03119428) was evaluated as a single drug, and two antibodies were combined with PD-1 blockers to evaluate MTIG7192A (NCT02794571) and BMS-986207 in some advanced primary brain tumours or other primary tumours (NCT02913313).

\section{VISTA}

Among tumour-infiltrating $\mathrm{T}$ cells and $\mathrm{M} 2$ macrophages (those macrophages that decrease inflammation and encourage tissue repair) in patients with localized and metastatic prostate cancer treated with ipilimumab, VISTA, PD-1 and PD-L1 have been shown to be co-upregulated. To date, there has been one terminated clinical trial utilizing an anti-VISTA monoclonal antibody (JNJ61610588) in advanced cancers (NCT02671955).

\section{COMBINATION IMMUNE CHECKPOINT INHIBITION}

Inhibitors of PD-1/PD-L1 have been shown to be effective in the treatment of many cancer types. However, it has been recently reported that in the process of inhibiting the PD-1/PD-L1 axis, the expression of other immune checkpoints, such as TIM-3, is increased, which may be related to adaptive resistance. ${ }^{81}$ Combined checkpoint inhibitors (CPIs) have been successfully used to improve tumour immune response and survival, and an increasing number of checkpoint inhibitors are targeting many costimulatory and cosuppressive interactions.

PD-1/CTLA4

In a clinical case report, for the first time, the combination of the anti-PD-1 antibody nivolumab $\left(3 \mathrm{mg} \cdot \mathrm{kg}^{-1}\right.$ body weight every 2 weeks) and the anti-CTLA4 antibody ipilimumab $\left(1 \mathrm{mg} \cdot \mathrm{kg}^{-1}\right.$ body weight every 6 weeks) was administered to a patient with HNSCC. $^{82}$ After 3 weeks of treatment with the drugs, computerized tomography (CT) examination of the patient showed a cancer response 4 months after surgery. However, magnetic resonance 
imaging (MRI) showed local recurrence after 7 months. The expression of PD-L1 and CTLA-4 significantly decreased. There was no significant change in PD-L2 expression or the numbers of $B$ cells, T cells, Th cells, cytotoxic and regulatory $\mathrm{T}$ lymphocytes, or NK cells. There are some ongoing clinical trials of the combination of PD-1 and CTLA-4 inhibitors in HNSCC (supplementary Table 2).

\section{PD-1/GITR}

Clinical data have shown that anti-GITR and anti-PD-1 antibody combination therapy can enhance the antitumour activity of $\mathrm{T}$ cells. ${ }^{83}$ BMS-986156 is a agonistic human $\operatorname{lgG} 1$ monoclonal antibody. It binds with GITR, promoting effector T cell activation, and may reduce or inactivate Treg cells. There has been a clinical phase I/lla study testing the role of BMS-986156 \pm nivolumab (anti-PD-1 mAb) in malignant solid tumours (NCT02598960).

In a dose-escalation study, 66 patients were asked to receive BMS-986156 (10-800 mg) or BMS-986156 (30-800 mg) with nivolumab $(240 \mathrm{mg})$ every 2 weeks. The results showed that BMS-986156 \pm nivolumab treatment was well tolerated. There was no obvious dose-limiting toxicity. Low immunogenicity and significant antitumour activity were observed with BMS-986156 and nivolumab at doses predicted to be biologically active. ${ }^{84}$

\section{PD-1/LAG-3}

Another phase I/lla dose-escalation and dose-expansion study, CA224-020, explored use of BMS-986016 (an anti-LAG3 antibody) as monotherapy and in combination with nivolumab for advanced solid tumours, including an HNSCC cohort.

Combinations with other therapies

Radiotherapy. It is estimated that more than $50 \%$ of patients with solid tumours receive radiotherapy (RT), which has an advantage over chemotherapy in that it limits systemic toxicity. ${ }^{85}$ In combination with immune checkpoint inhibitors, RT normalizes the tumour vascular system, enhances the expression of leukocyte adhesion molecules on endothelial cells, and results in secretion of $\mathrm{CD}^{+}$T-attracting chemokines. RT triggers immunogenic cell death by inducing cell surface exposure of calreticulin (CALR) and ATP secretion via autophagy and IFN-l-induced upregulation of MHC-I, HSP70 exposure, TLR3 signal, high-mobility group box 1 (HMGB1) release and $\mathrm{IL}-1 \beta$ release. ${ }^{86} \mathrm{RT}$ can induce signals to suppress immune activation, promote infiltration of bone marrowderived suppressor cells and lead to upregulation of PD-L1. ${ }^{87}$ As RT has certain vaccine characteristics, the combined use of RT with immune checkpoint inhibitors can produce synergistic effects. After receiving $\mathrm{RT}$, some patients may show somatic mutations that produce new antigens, which may become targets for stronger immune responses. The simultaneous use of RT and immune checkpoint inhibitors is considered safe, and immune-related adverse events have not been increased significantly. It is worth considering that high-dose radiotherapy may limit effective tumour immunity by reducing the production of IFN-I in irradiated tumours, whereas low-dose radiotherapy may cause the lymphocyte pool to shrink and eventually render immunotherapy ineffective.

Chemotherapy. Currently, all tumour-related antibodies are used in combination with chemotherapeutic drugs because they are less effective as monotherapy. ${ }^{88}$ Chemotherapy can assist immune checkpoint inhibitor treatment by releasing neoantigens or reshaping the tumour microenvironment by depleting Tregs and MDSCs. Chemotherapy can assist immunotherapy by downregulating PD-L2 expression on DCs and tumour cells, inducing APCs to mature, restoring tumour visibility, and upregulating MHC-I expression. ${ }^{89}$ Pemetrexed, carboplatin chemotherapy and pembrolizumab are approved therapies. Alterations to $\mathrm{T}$ cellrelated genes, such as upregulation of the IFN- $\gamma$ pathway and IFN$\mathrm{I}$, are involved in recruitment and activation. ${ }^{90}$ Pembrolizumab has no overlapping toxicity with these drugs. A phase II study evaluated the effectiveness of a combination of ipilimumab, paclitaxel and carboplatin in patients with stage IV non-small-cell lung carcinoma (NCT02279732). ${ }^{77}$ In addition, a comparative study of nivolumab combined with ipilimumab as the standard of care (extreme regimen) for first-line treatment of HNSCC is ongoing (NCT02741570). However, chemotherapy may cause lymphopenia and neutropenia, which may interfere with the mechanism of checkpoint inhibitors by inhibiting the clonal expansion of effector lymphocytes. Therefore, chemotherapy should be carefully considered.

Combinations with small molecule drugs (BRAF/TKs). Small molecule drugs are usually signalling inhibitors that can specifically block the signalling pathways necessary for tumour growth and proliferation, thereby achieving the purpose of treatment.

In $\sim 5 \%$ of human malignancies, BRAF oncogene mutations cause dysregulation, resulting in structural activation of the mitogen-activated protein kinase (MAPK) pathway and activation of mitogen-activated protein kinase (MEK). ${ }^{91}$ The activation of $B R A F$ can lead to the expression of anti-inflammatory cytokines and inhibit the function of TILs. The upregulation of PD-L1 is related to the formation of resistance to BRAF inhibitors. ${ }^{92} \mathrm{~A}$ phase $\mathrm{lb}$ trial demonstrated the use of BRAF and MEK inhibitors (cobimetinib and vemurafenib) in combination with atezolizumab (anti-PD-L1) in patients with metastatic melanoma with the BRAF V600E mutation. Triple therapy improved clinical efficacy and extended survival. ${ }^{93}$ In addition, there was a phase I trial comparing the safety and tolerability of durvalumab (MEDI4736) in combination with dabrafenib (BRAF inhibitor) and trametinib (BRAF inhibitor) with those of durvalumab in combination with trametinib (MEK inhibitor) alone (NCT02027961). A clinical trial of ipilimumab with or without dabrafenib, trametinib or nivolumab in patients with metastatic or unresectable melanoma is ongoing (NCT01940809).

Tyrosine kinases (TKs) have vital functions in growth factor signal transduction. Activated TKs can promote tumour cell proliferation, anti-apoptosis mechanisms, angiogenesis and metastasis. ${ }^{94}$ Sunitinib is a cellular signalling inhibitor that targets multiple tyrosine kinase receptors, including platelet-derived growth factors (PDGFRs), vascular endothelial growth factor receptors (VEGFRs) and c-KIT. ${ }^{95}$ A phase III clinical trial showed that pembrolizumab and avelumab in combination with the multi-TK inhibitor axitinib can benefit patients with renal cell carcinoma. ${ }^{96}$ Small molecules targeting c-KIT can reduce immunosuppressive MDSCs and show good activity when combined with anti-PD-1 or anti-CTLA-4 antibodies. The small molecule drug IPI-549 selectively inhibits the PI3K signalling pathway, which is highly expressed on myeloid cells and promotes migration in murine models of breast carcinoma and melanoma. ${ }^{97}$

Cancer Vaccines. Cancer vaccines have antigenicity and immunogenicity. For example, DC vaccines induce cancer-specific immune responses by carrying neoantigens encoded in DNA or mRNA or specific cell lysates. ${ }^{98}$ However, cancer vaccines do not combat the suppression of the tumour microenvironment, and studies found that molecules binding to immune checkpoint inhibitors on activated exhausted T cells could improve treatment outcomes. Using dual anti-CTLA-4/anti-PD-1 inhibitors and a DNA vaccine in mouse melanoma could increase the infiltration of $\mathrm{CD8}^{+} \mathrm{T}$ cells into the tumour. ${ }^{99}$ Currently, several clinical trials evaluating mRNA cancer vaccines are being conducted in combination with immune checkpoint inhibitors (NCT03633110, supplementary Table 2).

\section{CONCLUSIONS}

Immunotherapy is a promising approach to the treatment of patients with HNSCC. Both single-drug therapy and combination therapy have been shown to reduce morbidity and prolong the survival of patients with carcinoma. However, compared with 
conventional chemoradiotherapy, many immunotherapies take longer to achieve a clinical response and may even lead to tumour pseudoprogression. Differences in dose sequence and timing and in drug combinations may affect the magnitude and duration of immune-mediated antitumour activity. Therefore, as the understanding of the process of immune tumour cell death continues to deepen, guidelines will become available for the development of comprehensive treatment methods that enhance antitumour immunity and the sensitivity of tumour tissues to effector cell killing. ${ }^{100}$

However, we are still in the early stages of understanding the potential of immunotherapy and know little about the best way to combine surgery, chemotherapy, and radiotherapy with immunotherapy. Recently, upregulation of PD-L1 has been demonstrated in cancers treated with chemotherapy. This may indicate a potential benefit of the combined use of immunotherapy, chemotherapy and vaccines in the treatment of cancers. ${ }^{101}$

In addition, there are many challenges that need to be overcome to realize the clinical effects of immunotherapy: the choice of patients, the need for predictive biomarkers, and the need to test the relative efficacy of several immunotherapies over traditional drugs. In short, scientists still need to perform more investigations to achieve ideal treatments for clinical use to improve the survival of patients with HNSCC.

\section{ACKNOWLEDGEMENTS}

We thank Tian Wang for language editing.

\section{AUTHOR CONTRIBUTIONS}

Z.M. and J.H. are graduate students and contributed to the arrangement of all content, tables, and figures in this paper. B.Q. and A.K.-y.L. were responsible for the overall planning and editing of the manuscript.

\section{ADDITIONAL INFORMATION}

The online version of this article (https://doi.org/10.1038/s41368-020-0084-8) contains supplementary material, which is available to authorized users.

Competing interests: The authors declare no competing interests.

\section{REFERENCES}

1. Bray, F. et al. Global cancer statistics 2018: GLOBOCAN estimates of incidence and mortality worldwide for 36 cancers in 185 countries. CA Cancer J. Clin. 68, 394-424 (2018).

2. Pulte, D. \& Brenner, H. Changes in survival in head and neck cancers in the late 20th and early 21 st century: a period analysis. Oncologist 15, 994-1001 (2010).

3. Kuss, I., Hathaway, B., Ferris, R., Gooding, W. \& Whiteside, T. Decreased absolute counts of $\mathrm{T}$ lymphocyte subsets and their relation to disease in squamous cell carcinoma of the head and neck. Clin. Cancer Res. 10, 3755-3762 (2004).

4. Nordfors, C. et al. CD8+ and CD4+ tumour infiltrating lymphocytes in relation to human papillomavirus status and clinical outcome in tonsillar and base of tongue squamous cell carcinoma. Eur. J. Cancer 49, 2522-2530 (2013).

5. Fridman, W., Pagès, F., Sautès-Fridman, C. \& Galon, J. The immune contexture in human tumours: impact on clinical outcome. Nat. Rev. Cancer 12, 298-306 (2010).

6. Lee, M. H. et al. Interleukin 17 and peripheral IL-17-expressing T cells are negatively correlated with the overall survival of head and neck cancer patients. Oncotarget 9, 9825-9837 (2019).

7. López-Albaitero, A. et al. Role of antigen-processing machinery in the in vitro resistance of squamous cell carcinoma of the head and neck cells to recognition by CTL. J. Immunol. 176, 3402-3409 (2006).

8. Mizukami, Y. et al. Downregulation of HLA Class I molecules in the tumour is associated with a poor prognosis in patients with oesophageal squamous cell carcinoma. Br. J. Cancer 99, 1462-1467 (2008).

9. Ferris, R. L. Immunology and Immunotherapy of Head and Neck Cancer. J. Clin. Oncol. 33, 3293-3304 (2015).
10. Ferris, R. L., Whiteside, T. L. \& Ferrone, S. Immune escape associated with functional defects in antigen-processing machinery in head and neck cancer. Clin. Cancer Res. 12, 3890-3895 (2016).

11. Zou, W. \& Chen, L. Inhibitory B7-family molecules in the tumour microenvironment. Nat. Rev. Immunol. 8, 467-477 (2008).

12. Wang, X. B. et al. CTLA4 is expressed on mature dendritic cells derived from human monocytes and influences their maturation and antigen presentation. BMC immunol. 12, 21 (2011).

13. Jie, H. B. et al. CTLA-4 ${ }^{+}$Regulatory T Cells Increased in cetuximab-treated head and neck cancer patients suppress nk cell cytotoxicity and correlate with poor prognosis. Cancer Res. 75, 2200-2210 (2015).

14. Sullivan, T. J. et al. Lack of a role for transforming growth factor-beta in cytotoxic T lymphocyte antigen-4-mediated inhibition of T cell activation. Proc. Natl Acad. Sci. USA 98, 2587-2592 (2001).

15. Wing, J. B., Ise, W., Kurosaki, T. \& Sakaguchi, S. Regulatory T cells control antigenspecific expansion of Tfh cell number and humoral immune responses via the coreceptor CTLA-4. Immunity 41, 1013-1025 (2014).

16. Qureshi, O. S. et al. Constitutive clathrin-mediated endocytosis of CTLA-4 persists during T cell activation. J. Biol. Chem. 287, 9429-9440 (2012).

17. De Vicente, J. C. et al. PD-L1 expression in tumor cells is an independent unfavorable prognostic factor in oral squamous cell carcinoma. Cancer Epidemiol. Biomark. Prev. 28, 546-554 (2019).

18. Patel, S. P. \& Kurzrock, R. PD-L1 expression as a predictive biomarker in cance immunotherapy. Mol. Cancer Ther. 14, 847-856 (2015).

19. Hino, R. et al. Tumor cell expression of programmed cell death- 1 ligand 1 is a prognostic factor for malignant melanoma. Cancer 116, 1757-1766 (2010).

20. Wang, A. et al. The prognostic value of PD-L1 expression for non-small cell lung cancer patients: a meta-analysis. Eur. J. Surg. Oncol. 41, 450-456 (2015).

21. Zandberg, D. P. et al. The role of the PD-L1:PD-1 pathway in squamous cell carcinoma of the head and neck. Oral Oncol. 50, 627-632 (2014).

22. Lyford-Pike, S. et al. Evidence for a role of the PD-1:PD-L1 pathway in immune resistance of HPV-associated head and neck squamous cell carcinoma. Cancer Res. 73, 1733-1741 (2013).

23. Wang, W. et al. PD1 blockade reverses the suppression of melanoma antigenspecific CTL by CD4+ CD25(Hi) regulatory T cells. Int. Immunol. 21, 1065-1077 (2009).

24. Freeman, G. J. et al. Engagement of the PD-1 immunoinhibitory receptor by a novel B7 family member leads to negative regulation of lymphocyte activation. J. Exp. Med. 192, 1027-1034 (2000).

25. Monney, L. et al. Th1-specific cell surface protein Tim-3 regulates macrophage activation and severity of an autoimmune disease. Nature 415, 536-541 (2002).

26. Anderson, A. C. et al. Promotion of tissue inflammation by the immune receptor Tim-3 expressed on innate immune cells. Science 318, 1141-1143 (2007).

27. Zhou, Q. et al. Coexpression of Tim-3 and PD-1 identifies a CD8+ T-cell exhaustion phenotype in mice with disseminated acute myelogenous leukemia. Blood 117, 4501-4510 (2011).

28. Golden-Mason, L. et al. Galectin-9: Diverse roles in hepatic immune homeostasis and inflammation. Hepatology 66, 271-279 (2017).

29. Ngiow, S. F. et al. Anti-TIM3 antibody promotes T cell IFN- $\gamma$-mediated antitumor immunity and suppresses established tumors. Cancer Res. 71, 3540-3551 (2011).

30. Farren, M. R. et al. Systemic immune activity predicts overall survival in treatment-naïve patients with metastatic pancreatic cancer. Clin. Cancer Res. 22, 2565-2574 (2016).

31. Zheng, H. et al. Distinct role of Tim-3 in systemic lupus erythematosus and clear cell renal cell carcinoma. Int J. Clin. Exp. Med. 8, 7029-7038 (2015).

32. Liu, J. F. et al. T-cell immunoglobulin mucin 3 blockade drives an antitumor immune response in head and neck cancer. Mol. Oncol. 11, 235-247 (2017).

33. Finn, O. J. Immuno-oncology: understanding the function and dysfunction of the immune system in cancer. Ann. Oncol. 23, viii6-viii9 (2012).

34. Frisancho-Kiss, S. et al. Gonadectomy of male BALB/C mice increases Tim-3(+) alternatively activated M2 macrophages, Tim-3(+) T cells, Th2 cells and Treg in the heart during acute coxsackievirus-induced myocarditis. Brain Behav. Immun. 23, 649-657 (2009).

35. Triebel, F. et al. LAG-3, a novel lymphocyte activation gene closely related to CD4. J. Exp. Med. 171, 1393-1405 (1990).

36. Huard, B. et al. Lymphocyte-activation gene $3 /$ major histocompatibility complex class II interaction modulates the antigenic response of CD4+ T lymphocytes. Eur. J. Immunol. 24, 3216-3221 (1994).

37. Workman, C. J. \& Vignali, D. A. The CD4-related molecule, LAG-3 (CD223), regulates the expansion of activated T cells. Eur. J. Immunol. 33, 970-979 (2003).

38. Huang, C. T. et al. Role of LAG-3 in regulatory T cells. Immunity 21, 503-513 (2004)

39. Woo, S. R. et al. Immune inhibitory molecules LAG-3 and PD-1 synergistically regulate T-cell function to promote tumoral immune escape. Cancer Res. 72, 917-927 (2012). 
40. Huard, B. et al. CD4/major histocompatibility complex class II interaction analyzed with CD4- and lymphocyte activation gene-3 (LAG-3)-Ig fusion proteins. Eur. J. Immunol. 25, 2718-2721 (1995).

41. Xu, F. et al. LSECtin expressed on melanoma cells promotes tumor progression by inhibiting antitumor T-cell responses. Cancer Res. 74, 3418-3428 (2014).

42. Matsuzaki, J. et al. Tumor-infiltrating NY-ESO-1-specific CD8+ T cells are negatively regulated by LAG-3 and PD-1 in human ovarian cancer. Proc. Natl Acad. Sci. USA 107, 7875-7880 (2010).

43. Grosso, J. F. et al. LAG-3 regulates CD8+ T cell accumulation and effector function in murine self- and tumor-tolerance systems. J. Clin. Invest. 117, 3383-3392 (2007).

44. Jie, H. B. et al. Intratumoral regulatory $T$ cells upregulate immunosuppressive molecules in head and neck cancer patients. Br. J. Cancer 109, 2629-2635 (2013).

45. Wang, J. et al. Fibrinogen-like Protein 1 is a major immune inhibitory ligand of LAG-3. Cell 176, 334-347 (2019).

46. Demchev, V. et al. Targeted deletion of fibrinogen like protein 1 reveals a novel role in energy substrate utilization. PLoS One 8, e58084 (2013).

47. $\mathrm{Yu}, \mathrm{X}$. et al. The surface protein TIGIT suppresses $\mathrm{T}$ cell activation by promoting the generation of mature immunoregulatory dendritic cells. Nat. Immunol. 10, 48-57 (2009)

48. Fuchs, A. \& Colonna, M. The role of NK cell recognition of nectin and nectin-like proteins in tumor immunosurveillance. Semin Cancer Biol. 16, 359-366 (2006).

49. Lozano, E., Dominguez-Villar, M., Kuchroo, V. \& Hafler, D. A. The TIGIT/CD226 axis regulates human T cell function. J. Immunol. 188, 3869-3875 (2012).

50. Stephens, G. L. et al. Engagement of glucocorticoid-induced TNFR family-related receptor on effector $T$ cells by its ligand mediates resistance to suppression by CD4+CD25+ T cells. J. Immunol. 173, 5008-5020 (2004).

51. Schaer, D. A. et al. GITR pathway activation abrogates tumor immune suppression through loss of regulatory $\mathrm{T}$ cell lineage stability. Cancer Immunol. Res. 1, 320-331 (2013).

52. Esparza, E. M. \& Arch, R. H. Glucocorticoid-induced TNF receptor functions as a costimulatory receptor that promotes survival in early phases of T cell activation. J. Immunol. 174, 7869-7874 (2015).

53. Shimizu, J. et al. Stimulation of CD25(+)CD4(+) regulatory T cells through GITR breaks immunological self-tolerance. Nat. Immunol. 3, 135-142 (2002).

54. Le Mercier, I. et al. VISTA Regulates the development of protective antitumor immunity. Cancer Res. 74, 1933-1944 (2014).

55. Wang, L. et al. VISTA, a novel mouse Ig superfamily ligand that negatively regulates T cell responses. J. Exp. Med. 208, 577-592 (2011).

56. Liu, J. et al. Immune-checkpoint proteins VISTA and PD-1 nonredundantly regulate murine T-cell responses. Proc. Natl Acad. Sci. USA 112, 6682-6687 (2015).

57. Wang, J. et al. VSIG-3 as a ligand of VISTA inhibits human T-cell function. Immunology 156, 74-85 (2019).

58. Harada, H., Suzu, S., Hayashi, Y. \& Okada, S. BT-IgSF, a novel immunoglobulin superfamily protein, functions as a cell adhesion molecule. J. Cell Physiol. 204, 919-926 (2015)

59. Topalian, S. L. et al. Safety, activity, and immune correlates of anti-PD-1 antibody in cancer. N. Engl. J. Med. 366, 2443-2454 (2012).

60. Balar, A. V. \& Weber, J. S. PD-1 and PD-L1 antibodies in cancer: current status and future directions. Cancer Immunol. Immunother. 66, 551-564 (2017).

61. Kaidar-Person, O., Gil, Z. \& Billan, S. Precision therapy of head and neck squamous cell carcinoma. J. Dent. Res. 40, 13-16 (2018).

62. Ferris, R. L. et al. Nivolumab for recurrent squamous-cell carcinoma of the head and neck. N. Engl. J. Med. 375, 1856-1867 (2016).

63. Harrington, K. J. et al. Nivolumab versus standard, single-agent therapy of investigator's choice in recurrent or metastatic squamous cell carcinoma of the head and neck (CheckMate 141): health-related quality-of-life results from a randomised, phase 3 trial. Lancet Oncol. 18, 1104-1115 (2017).

64. Seiwert, T. Y. et al. Safety and clinical activity of pembrolizumab for treatment of recurrent or metastatic squamous cell carcinoma of the head and neck (KEYNOTE012): an open-label, multicentre, phase 1b trial. Lancet Oncol. 17, 956-965 (2017).

65. Burtness, B. et al. Pembrolizumab alone or with chemotherapy versus cetuximab with chemotherapy for recurrent or metastatic squamous cell carcinoma of the head and neck (KEYNOTE-048): a randomised, open-label, phase 3 study. Lancet 394, 1915-1928 (2019).

66. Festino, L. et al. Cancer treatment with anti-PD-1/PD-L1 agents: Is PD-L1 expression a biomarker for patient selection? Drugs 6, 925-945 (2016).

67. Chow, L. Q. et al. Antitumor activity of pembrolizumab in biomarker-unselected patients with recurrent and/or metastatic head and neck squamous cell carcinoma: results from the phase Ib KEYNOTE-012 Expansion Cohort. J. Clin. Oncol. 34, 3838-3845 (2016).

68. Zandberg, D. P. et al. Durvalumab for recurrent or metastatic head and neck squamous cell carcinoma: Results from a single-arm, phase II study in patients with $\geq 25 \%$ tumour cell PD-L1 expression who have progressed on platinumbased chemotherapy. Eur. J. Cancer 107, 142-152 (2018).
69. Taube, J. M. et al. Association of PD-1, PD-1 ligands, and other features of the tumor immune microenvironment with response to anti-PD-1 therapy. Clin. Cancer Res 20, 5064-5074 (2014).

70. Yearley, J. H. et al. PD-L2 expression in human tumors: relevance to anti-PD-1 therapy in cancer. Clin. Cancer Res 23, 3158-3167 (2017).

71. Zhang, J. et al. Co-expression of PD-1 and PD-L1 predicts poor outcome in nasopharyngeal carcinoma. Med. Oncol. 32, 86 (2015).

72. Lee, V. H. et al. Correlation of PD-L1 expression of tumor cells with survival outcomes after radical intensity-modulated radiation therapy for non-metastatic nasopharyngeal carcinoma. PLoS One. 11, e0157969 (2016).

73. Blank, C. U. Therapeutic use of anti-CTLA-4 antibodies. Int. Immunol. 27, 3-10 (2015).

74. Camacho, L. H. et al. Phase I/II trial of tremelimumab in patients with metastatic melanoma. J. Clin. Oncol. 27, 1075-1081 (2009).

75. Hamid, O. et al. A prospective phase II trial exploring the association between tumor microenvironment biomarkers and clinical activity of ipilimumab in advanced melanoma. J. Transl. Med. 9, 204 (2011).

76. Reck, M. et al. Ipilimumab in combination with paclitaxel and carboplatin as first-line therapy in extensive-disease-small-cell lung cancer: results from a randomized, double-blind, multicenter phase 2 trial. Ann. Oncol. 24, 75-83 (2013).

77. Lynch, T. J. et al. Ipilimumab in combination with paclitaxel and carboplatin as first-line treatment in stage IIIB/IV non-small-cell lung cancer: results from a randomized, double-blind, multicenter phase II study. J. Clin. Oncol. 30, 2046-2054 (2012).

78. Tran, B. et al. Dose escalation results from a first-in-human, phase 1 study of glucocorticoid-induced TNF receptor-related protein agonist AMG 228 in patients with advanced solid tumors. J. Immunother. Cancer 6, 93 (2018).

79. Kim, J. E. et al. Combination therapy with anti-PD-1, Anti-TIM-3, and focal radiation results in regression of murine gliomas. Clin. Cancer Res. 23, 124-136 (2017).

80. Ascierto, P. A. et al. Initial efficacy of anti-lymphocyte activation gene-3 (anti-LAG-3; BMS-986016) in combination with nivolumab (nivo) in pts with melanoma (MEL) previously treated with anti-PD-1/PD-L1 therapy. J. Clin. Oncol. 35, 9520 (2017).

81. Koyama, S. et al. Adaptive resistance to therapeutic PD-1 blockade is associated with upregulation of alternative immune checkpoints. Nat. Commun. 7, 10501 (2016).

82. Schwab, K. S. et al. Successful treatment of refractory squamous cell cancer of the head and neck with nivolumab and ipilimumab. Case Rep. Oncol. 11, 17-20 (2018).

83. Shevach, E. M. \& Stephens, G. L. The GITR-GITRL interaction: co-stimulation or contrasuppression of regulatory activity? Nat. Rev. Immunol. 6, 613-618 (2006).

84. Siu, L. L. et al. Preliminary results of a phase I/lla study of BMS-986156 (glucocorticoid-induced tumor necrosis factor receptor-related gene [GITR] agonist), alone and in combination with nivolumab in pts with advanced solid tumors. J. Clin. Oncol. 35, 104-104 (2017).

85. Trommer, M. et al. Abscopal effects in radio-immunotherapy-response analysis of metastatic cancer patients with progressive disease under anti-PD-1 immune checkpoint inhibition. Front Pharmacol. 10, 511 (2019).

86. Ko, E. C. \& Formenti, S. C. Radiation therapy to enhance tumor immunotherapy: a novel application for an established modality. Int. J. Radiat. Biol. 95, 936-939 (2019).

87. Zappasodi, R., Merghoub, T. \& Wolchok, J. D. Emerging concepts for immune checkpoint blockade-based combination therapies. Cancer Cell. 34, 581-598 (2018).

88. Cruz, E. \& Kayser, V. Monoclonal antibody therapy of solid tumors: clinical limitations and novel strategies to enhance treatment efficacy. Biologics 13, 33-51 (2019).

89. Qiao, M., Jiang, T., Ren, S. \& Zhou, C. Combination strategies on the basis of immune checkpoint inhibitors in non-small-cell lung cancer: where do we stand? Clin. Lung Cancer 19, 1-11 (2018).

90. Garassino, M. C., Torri, V., Colombo, M. P. \& Sica, A. Choosing the best chemotherapy agent to boost immune checkpoint inhibition activity. Cancer Res. 78, 5729-5730 (2018).

91. Morris, V. \& Kopetz, S. BRAF inhibitors in clinical oncology. F1000Prime Rep. 5, 11 (2013).

92. Sullivan, R. J. et al. Atezolizumab plus cobimetinib and vemurafenib in BRAFmutated melanoma patients. Nat. Med. 25, 929-935 (2019).

93. Amaria, R. N. et al. Neoadjuvant plus adjuvant dabrafenib and trametinib versus standard of care in patients with high-risk, surgically resectable melanoma: a single-centre, open-label, randomised, phase 2 trial. Lancet Oncol. 19, 181-193 (2018).

94. Kuusk, T. et al. Antiangiogenic therapy combined with immune checkpoint blockade in renal cancer. Angiogenesis 20, 205-215 (2017).

95. Choueiri, T. K. \& Motzer, R. J. Systemic therapy for metastatic renal-cell carcinoma. N. Engl. J. Med. 376, 354-366 (2017). 
96. Caruso, C. Checkpoint Inhibitor-TKI Combos Effective in RCC. Cancer Discov 9, 460 (2019).

97. De Henau, O. et al. Overcoming resistance to checkpoint blockade therapy by targeting PI3Ky in myeloid cells. Nature 539, 443-447 (2016).

98. Prins, R. M. et al. Comparison of glioma-associated antigen peptide-loaded versus autologous tumor lysate-loaded dendritic cell vaccination in malignant glioma patients. J. Immunother. 36, 152-157 (2013).

99. Kos, S. et al. Intradermal DNA vaccination combined with dual CTLA-4 and PD-1 blockade provides robust tumor immunity in murine melanoma. PloS One 14, e0217762 (2019).

100. Cruickshank, B. et al. Dying to be noticed: epigenetic regulation of immunogenic cell death for cancer immunotherapy. Front Immunol. 9, 654 (2018).

101. $\mathrm{Ng}, \mathrm{H}$. Y. et al. Chemotherapeutic treatments increase PD-L1 expression in esophageal squamous cell carcinoma through EGFR/ERK activation. Transl. Oncol. 11, 1323-1333 (2018).
Open Access This article is licensed under a Creative Commons Attribution 4.0 International License, which permits use, sharing, adaptation, distribution and reproduction in any medium or format, as long as you give appropriate credit to the original author(s) and the source, provide a link to the Creative Commons license, and indicate if changes were made. The images or other third party material in this article are included in the article's Creative Commons license, unless indicated otherwise in a credit line to the material. If material is not included in the article's Creative Commons license and your intended use is not permitted by statutory regulation or exceeds the permitted use, you will need to obtain permission directly from the copyright holder. To view a copy of this license, visit http://creativecommons. org/licenses/by/4.0/.

(c) The Author(s) 2020 